\title{
Genetic disruption of the oncogenic HMGA2-PLAG1-IGF2 pathway causes fetal growth restriction
}

\author{
Walid Abi Habib, $\mathrm{PhD}^{1,2,10}$, Frédéric Brioude, $\mathrm{MD}, \mathrm{PhD}^{1,2}$, Thomas Edouard, $\mathrm{MD}, \mathrm{PhD}^{3,4}$, \\ James T. Bennett, MD, PhD ${ }^{5}$, Anne Lienhardt-Roussie, MD, PhD ${ }^{6}$, Frédérique Tixier, $\mathrm{MD}^{7}$, \\ Jennifer Salem, $\mathrm{MSc}^{8}$, Tony Yuen, $\mathrm{PhD}^{9}$, Salah Azzi, $\mathrm{PhD}^{1,2}$, Yves Le Bouc, MD, PhD ${ }^{1,2}$, \\ Madeleine $\mathrm{D}$. Harbison, $\mathrm{MD}^{9}$ and Irène Netchine, $\mathrm{MD}, \mathrm{PhD}^{1,2}$
}

\begin{abstract}
Purpose: Fetal growth is a complex process involving maternal, placental and fetal factors. The etiology of fetal growth retardation remains unknown in many cases. The aim of this study is to identify novel human mutations and genes related to Silver-Russell syndrome (SRS), a syndromic form of fetal growth retardation, usually caused by epigenetic downregulation of the potent fetal growth factor IGF2.

Methods: Whole-exome sequencing was carried out on members of an SRS familial case. The candidate gene from the familial case and two other genes were screened by targeted high-throughput sequencing in a large cohort of suspected SRS patients. Functional experiments were then used to link these genes into a regulatory pathway.

Results: We report the first mutations of the PLAG1 gene in humans, as well as new mutations in HMGA2 and IGF2 in six
\end{abstract}

sporadic and/or familial cases of SRS. We demonstrate that HMGA2 regulates IGF2 expression through PLAG1 and in a PLAG1-independent manner.

Conclusion: Genetic defects of the HMGA2-PLAG1-IGF2 pathway can lead to fetal and postnatal growth restriction, highlighting the role of this oncogenic pathway in the fine regulation of physiological fetal/postnatal growth. This work defines new genetic causes of SRS, important for genetic counseling.

Genet Med advance online publication 10 August 2017

Key Words: fetal growth restriction; HMGA2; IGF2; PLAG1; Silver-Russell syndrome

\section{INTRODUCTION}

Intrauterine growth retardation (IUGR) is a common condition arising from multiple origins (environmental, (epi)genetic, vascular, etc.). ${ }^{1} \mathrm{~A}$ well-characterized syndromic form of IUGR is represented by Silver-Russell syndrome (SRS, OMIM 180860). SRS is a clinically and genetically heterogeneous imprinting disorder characterized by fetal and postnatal growth retardation, relative macrocephaly at birth, a prominent forehead, and additional features. ${ }^{2}$ The syndrome remains a clinical diagnosis, defined by clinical scoring systems. ${ }^{2-6}$ Insulin-like growth factor 2 (IGF2) is a growth factor strongly implicated in fetal growth and in the pathophysiology of SRS, as up to $50-60 \%$ of patients display hypomethylation of the imprinted 11p15.5 IGF2/H19 domain, which leads to the downregulation of IGF2 expression. ${ }^{2,7,8}$ The second most common cause of SRS is maternal uniparental disomy of chromosome 7, accounting for about $10 \%$ of cases. ${ }^{2}$ Other rare 11 p15.5-related genetic defects, such as maternal duplications, maternal CDKN1C mutation, ${ }^{9}$ and paternal IGF2 point mutation ${ }^{10}$ have also been implicated in SRS. In about $30-40 \%$ of patients with a clinical diagnosis of SRS, the molecular etiology of this syndrome remains unknown, involving molecular mechanisms and genes other than those cited above. ${ }^{6}$

Pleomorphic adenoma gene 1 (PLAG1), on human chromosome $8 \mathrm{q} 12$, was initially identified as an oncogene associated with certain types of cancer (e.g., pleomorphic adenomas, lipoblastoma, hepatoblastoma). ${ }^{11-13}$ The tumorigenic capacity of PLAG1 results from its ability to bind the P3 promoter of IGF2, thereby increasing IGF2 expression in tumor cells. ${ }^{13-16}$ In addition to functioning as an oncogene, PLAG1 has been implicated in growth, as Plag1 knockout mice and paternal Igf2-deficient mice have remarkably similar phenotypes, characterized by intrauterine and postnatal growth retardation. ${ }^{17}$

The DNA-binding protein high-mobility group AT-hook 2 (HMGA2), located on human chromosome 12q14, is a transcription factor that has been identified as an oncogene

\footnotetext{
${ }^{1}$ Sorbonne Universités, UPMC Univ Paris 06, UMR_S 938, CDR Saint-Antoine, Paris, France; ${ }^{2}$ Service d’Explorations Fonctionnelles Endocriniennes, AP-HP, Hôpital Trousseau, Paris, France; ${ }^{3}$ Endocrine, Bone Diseases, and Genetics Unit, Children's Hospital, University Hospital Center, Toulouse, France; ${ }^{4}$ INSERM Unit 1043 , Physiopathology Center of Toulouse Purpan (CTPT), Paul-Sabatier University, Toulouse, France; ${ }^{5}$ Department of Pediatrics (Genetics), University of Washington, and Center for Integrative Brain Research, Seattle Children's Research Institute, Seattle, Washington, USA; ${ }^{6}$ Département de Pédiatrie Médicale, Centre Hospitalo-Universitaire de Limoges, Limoges Cedex, France;

${ }^{7}$ Département d'Endocrinologie Pédiatrique, Hôpital Debrousse, Lyon, France; ${ }^{8}$ RSS/SGA Research \& Education Fund, MAGIC Foundation, Oak Park, Illinois, USA; ${ }^{9}$ Department of Pediatrics, Icahn School of Medicine at Mount Sinai, New York, New York, USA; ${ }^{10}$ Current affiliation: Center for Epigenetics, Van Andel Research Institute, Grand Rapids, Michigan, USA. Correspondence: Irène Netchine (irene.netchine@aphp.fr)

The last three authors contributed equally to this work.

Submitted 16 March 2017; accepted 29 May 2017; advance online publication 10 August 2017. doi:10.1038/gim.2017.105
} 
and shown to be an upstream regulator of PLAG1 expression in several tumor cells and experimental models. ${ }^{18} \mathrm{Hmga2}$ deficient mice have a pygmy phenotype and fetal growth retardation. ${ }^{19}$ A splicing mutation of HMGA2 was recently associated with SRS in a family but the contribution of this mutation to the phenotype was not clearly assessed. ${ }^{20}$

Rearrangements of $8 \mathrm{q} 12$ and 12q14 chromosomal regions corresponding to the locations of PLAG1 and HMGA2, respectively, have been reported in patients with SRS and SRS-like conditions. ${ }^{21}$ Finally, GWAS meta-analysis studies on humans have shown HMGA2 and PLAG1 variants to be strongly correlated with childhood/adult height, highlighting the role of these genes in the control of human growth. ${ }^{22-25}$

Together, these observations strongly suggest that HMGA2 and PLAG1 play a role in growth physiology mediated by IGF2, but direct evidence has been lacking.

Here we report new mutations in the HMGA2-PLAG1IGF2 pathway resulting in lower levels of IGF2 expression in SRS patients. These findings highlight the role of HMGA2 and PLAG1 as upstream regulators of IGF2.

\section{Population studied}

MATERIALS AND METHODS

The familial case and the 192 patients included in this study were referred for IUGR and suspected Silver-Russell syndrome. Blood samples were collected during clinical visits.

Written informed consent for participation was received from all patients or parents, in accordance with national ethics rules (Assistance Publique-Hôpitaux de Paris authorization no. 681 for French patients and IRB I00000204 at the Mount Sinai School of Medicine, New York, for patients recruited in the United States). Written consent was also obtained to publish patient photos. Patients were either followed at Armand Trousseau Children's Hospital or referred by other clinical centers for molecular analysis of suspected SRS. A geneticist and/or a pediatric endocrinologist examined each patient and a detailed clinical form was completed. Patients who were negative for methylation defects within the imprinted domains controlling IGF2/H19, DLK1/GTL2, and MEST/ GRB10 assessed by allele-specific methylated multiplex realtime quantitative polymerase chain reaction, as previously described, ${ }^{2}$ were retained for further molecular analysis.

\section{Whole-exome sequencing and targeted}

\section{HMGA2-PLAG1-IGF2 sequencing}

Library preparation, exome capture/gene enrichment, sequencing, and data analysis were performed by IntegraGen SA (Evry, France). The sequencing methods and bioinformatics analysis are described in detail in the Supplementary Methods online.

\section{Sanger sequencing and short tandem repeat typing}

HMGA2, PLAG1, and IGF2 mutations identified with wholeexome sequencing and targeted sequencing were verified by standard methods of Sanger sequencing, using the ABI PRISM Big Dye Terminator v3.0 Cycle Sequencing Kit and an ABI 3100 Genetic Analyzer (Life Technologies, Courtaboeuf, France). The sequencing products were then analyzed with SeqScape v2.6 (Life Technologies). For de novo mutations, parental inheritance was verified using short tandem repeat typing on chromosome 14 (D14S65 and D14S292).

\section{Cell cultures and transfections}

Fibroblasts, Hep3B, and HEK293 cells were cultured under standard conditions in supplemented RPMI 1640 and MEM, respectively (Gibco, Cergy Pontoise, France), at $37^{\circ} \mathrm{C}$. Gene silencing and overexpression assays were performed according to the manufacturer's protocols (Thermo Fisher, France). Details of the culture conditions, gene silencing, and overexpression assays as well as RNA extraction are provided in the Supplementary Methods.

\section{Reverse transcription and real-time messenger RNA quantification}

We generated complementary DNA from the messenger RNA of fibroblasts, Hep3B, and HEK293 cells, with the SuperScript II reverse transcription system (\#18064-014 Invitrogen Thermo Fisher, France). Real-time PCR was performed on the complementary DNAs obtained, in an ABI-7900HT machine, and gene expression was quantified with the Power SYBR Green PCR Master Mix (Life Technologies). Details from the reverse transcription and real-time quantification analysis are provided in the Supplementary Methods.

\section{Statistical analysis}

We compared data for pairs of groups in Mann-Whitney (expression in fibroblasts) and unpaired $t$ (silencing and overexpressing assays) tests. We considered $P$ values of less than 0.05 to indicate statistical significance. The analyses were performed with GraphPad Prism version 6.00 (GraphPad Software, La Jolla, CA).

\section{RESULTS}

\section{Heterozygous PLAG1 frameshift mutation}

SRS was diagnosed clinically using the Netchine-Harbison clinical scoring system (NH-CSS $)^{2,6}$ in patient II-2 from the affected family (Figure 1). Molecular analysis revealed a normal methylation on chromosomes 11, 7, and 14. Singlenucleotide polymorphism array excluded maternal uniparental disomies and chromosomal rearrangements. The proband's sister and mother had similar phenotypes consistent with dominant transmission of the disease. Wholeexome sequencing revealed a heterozygous deletion of a single nucleotide within exon 5 of the PLAG1 gene (NM_002655.2: c.439del) in the proband, her mother, and her sister, but not in the father. This deletion leads to a frameshift and production of a truncated, 227-amino acid peptide (NP_002646.2:p.Ser147Valfs ${ }^{\star} 2$ ). This variant was not reported in any polymorphism or the ExAC database. The 18 other variants listed in Supplementary Table 1, which were found in the three affected family members, have no known effect on growth. 
PLAG1 mutations

I-2

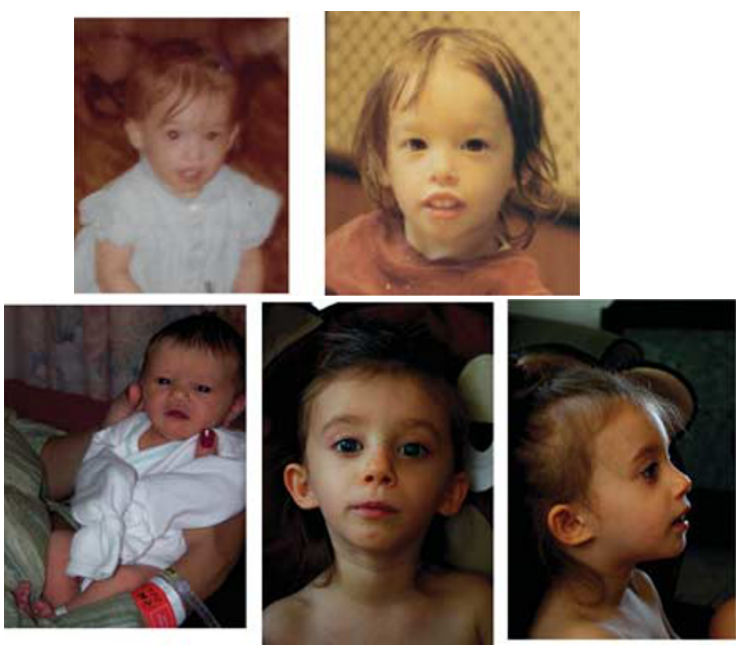

I
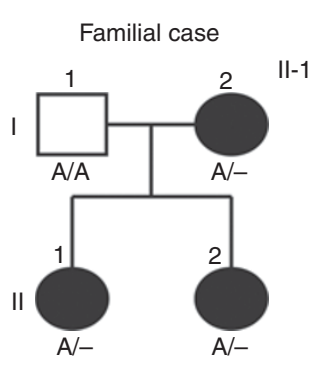

II-2
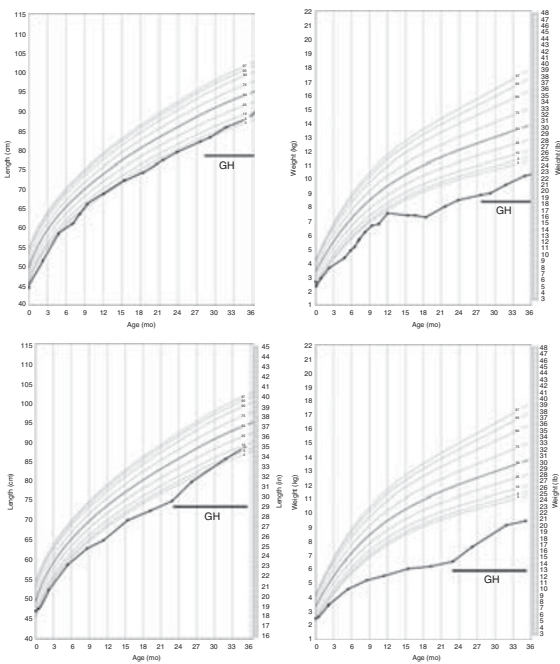

Sporadic case

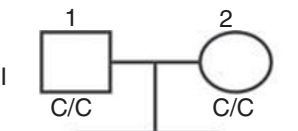

II

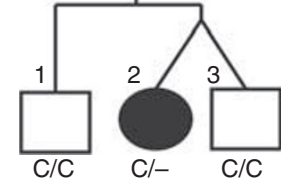

HMGA2 mutation

NM_003483.4:c. 193C>T
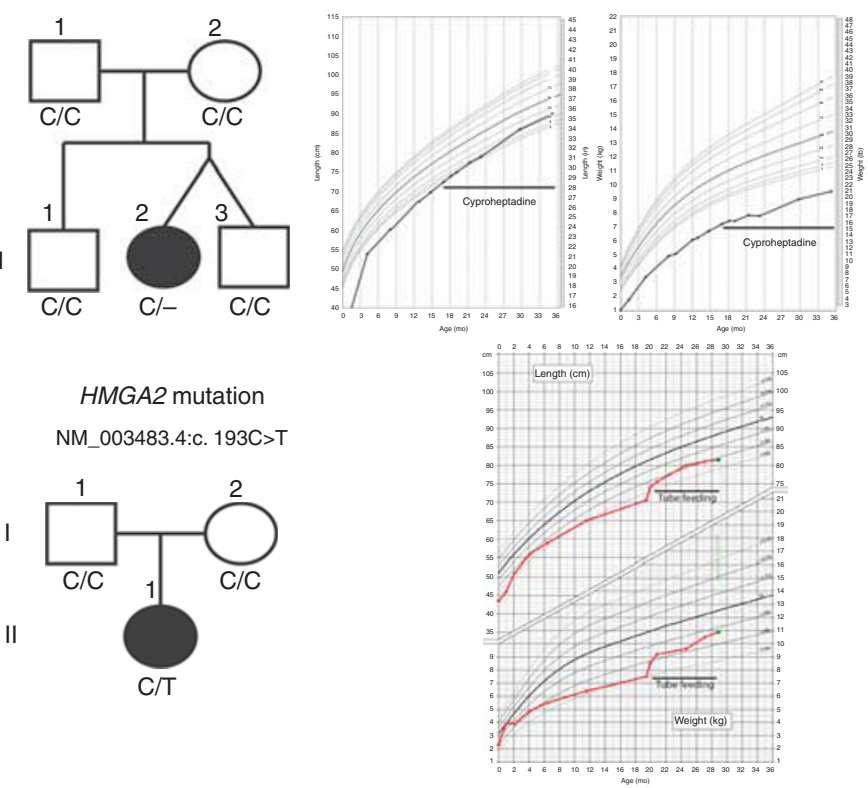

IGF2 mutation NM_000612.5:c. 78C>G

I

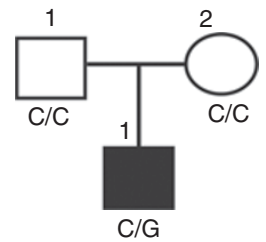

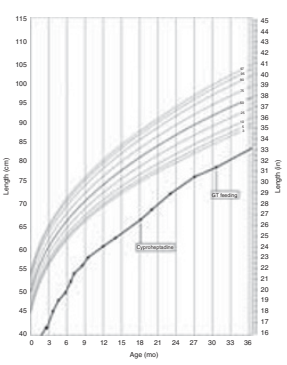

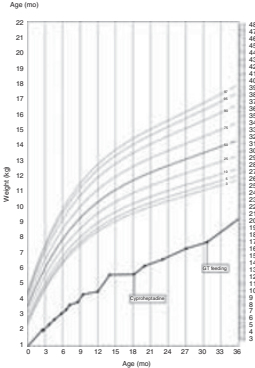

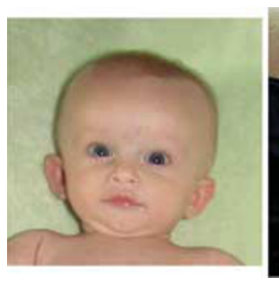
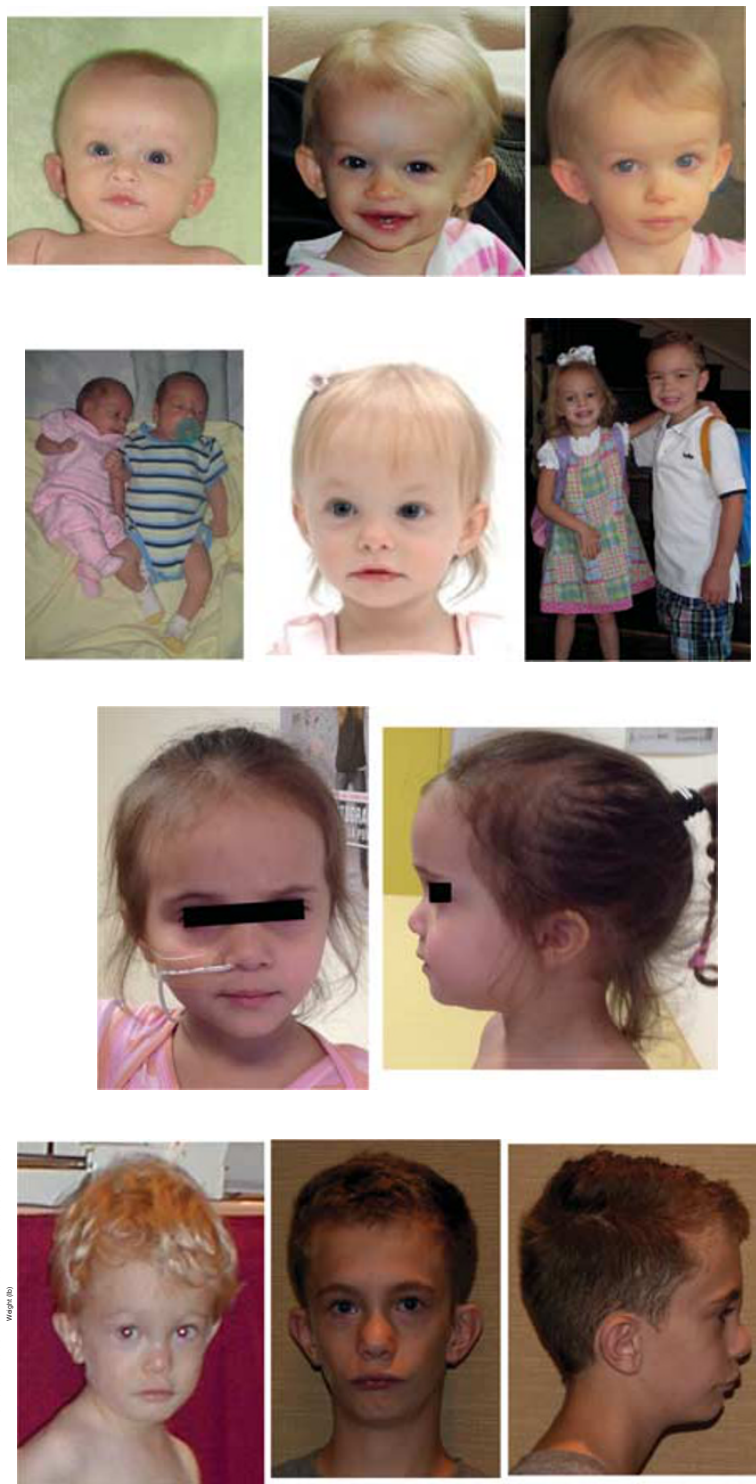

Figure 1 Pedigrees, growth charts, and photographs of patients carrying mutations affecting the HMGA2-PLAG1-IGF2 pathway. The patient with sporadic PLAG1 anomaly is next to her healthy twin brother. The patient with /GF2 anomaly shows that a prominent forehead evident in early childhood may become less obvious in later life. The patients' growth charts between the ages of 2 and 20 years are included in the Supplementary Figure. 


\section{Description of the screened population}

We have selected 192 patients for whom a diagnosis of SRS was suspected and for whom we could not identify any known epigenetic or genetic cause of SRS. All these patients were born small for gestational age (birth weight and/or birth length $<-2$ standard deviation score (SDS)) and without body asymmetry, which is in favor of a genetic rather than an epigenetic defect. Of these patients, 76 displayed head circumference sparing with relative macrocephaly at birth.

\section{Identification of new genetic defects affecting the HMGA2- PLAG1-IGF2 pathway}

As PLAG1 plays a key role in the HMGA2-PLAG1-IGF2 pathway, we screened for mutations using high-throughput targeted sequencing for these three genes in the cohort of patients described above. We identified another heterozygous, de novo deletion of a single nucleotide in PLAG1 (NM_002655.2:c.1363del), leading to a frameshift and the production of a truncated, 469-amino acid protein (NP_002646.2:pGln455Serfs $\left.{ }^{\star} 16\right)$. This deletion was not found in the parents, the twin brother, or the older brother of the patient (Figure 1). Two new heterozygous variants were identified in the HMGA2 gene: a de novo nonsense mutation (NM_003483.4:c.193C > T) leading to premature termination (NP_003474.1:p.Gln65*), and a deletion of a single nucleotide (NM_003483.4:c.189del) leading to an elongated protein (NP_003474.1:p.Ala64Leufs ${ }^{\star} 102$ ) (Table 1). Due to lack of parental DNA, the origin of this deletion could not be investigated, but the father's adult height of $155 \mathrm{~cm}$ strongly suggests that he passed this deletion to his son. Finally, we found two heterozygous variations within the IGF2 gene: a de novo nonsense mutation (NM_000612.5: c.78C $>\mathrm{G}$ ) leading to the production of a truncated peptide (NP_000603.1:p.Tyr26*), and a de novo two-nucleotide duplication (NM_000612.5:c.158_159dup) leading to a frameshift and the production of a truncated 59-amino acid protein (NP_000603.1:p.Arg54Alafs*7).

All five mutations were identified in the group of 76 patients with relative macrocephaly at birth.

\section{Clinical data}

The clinical presentation of the patients harboring the mutations is summarized in Table 1 . We were unable to categorize patient I-2 due to a lack of information about some of her clinical signs. For the remaining patients, five out of seven fulfilled the SRS clinical diagnostic criteria of the $\mathrm{NH}-\mathrm{CSS}$ (at least four of six criteria, including relative macrocephaly). Patient II-1 fulfilled only three of the six criteria from the NH-CSS and patient II-2 fulfilled four of six criteria without relative macrocephaly at birth. Birth weights and lengths were most strongly affected for patients with IGF2 and HMGA2 mutations (respectively), with a slightly smaller effect observed in patients with PLAG1 defects. Similarly, relative macrocephaly, defined as a difference in SDS of at least 1.5 between head circumference and length or weight at birth, was more pronounced in patients with the IGF2 mutations. As is characteristic of SRS, all patients presented with significant feeding difficulties and need for nutritional support with nonvolitional feeding and/or appetite stimulation. Serum IGF2 levels and other related markers from the patients with PLAG1, HMGA2, and IGF2 mutations are listed in Table 1.

\section{Regulation of IGF2 levels by HMGA2 and HMAG2-PLAG1 pathway}

The small interfering RNA (siRNA)-mediated silencing of HMGA2 in Hep3b cells decreased PLAG1 and total IGF2 expression (Figure 2a). Silencing of PLAG1 resulted in lower levels of total IGF2, but did not change the levels of HMGA2 expression (Figure 2a). Since PLAG1 binds specifically to the IGF2 promoter P3, we assessed the levels of IGF2 generated by transcription from the $\mathrm{P} 1$ and $\mathrm{P} 3$ promoters (IGF2-P1 and IGF2-P3, respectively). The silencing of both $H M G A 2$ and PLAG1 led to a downregulation of IGF2-P3, whereas IGF2-P1 expression remained normal (Figure 2b). Finally, H19 expression was not affected by the silencing of either HMGA2 or PLAG1. PLAG1 therefore acts as a trans-acting factor at the IGF2-P3 promoter, but does not affect the whole IGF2/H19 domain (Figure 2a). IGF2-P3 levels were significantly lower in siHMGA2-treated than in siPLAG1-treated cells despite the production of significantly lower levels of PLAG1 in siPLAG1-treated cells (Figure 2a,b), suggesting a PLAG1-independent HMGA2 regulation of IGF2. In order to verify this, we performed an overexpressing assay of HMGA2 and PLAG1 in HEK293 cells. Overexpression of HMGA2 in these cells resulted in an increased expression of IGF2-P3, without affecting the messenger RNA levels of PLAG1 (Figure 2c). However, PLAG1 maintained its capacity to upregulate IGF2-P3 upon PLAG1 overexpression (Figure 2c). These results show that HMGA2 and PLAG1 both positively regulate IGF2-P3 expression, independently or via a HMGA2PLAG1-IGF2 pathway.

We further investigated gene expression in fibroblasts from patient I-2 from the family (Figure 1) carrying the PLAG1 deletion. The levels of PLAG1 expression were similar to those in controls, but IGF2 expression was half that of the controls (Figure 2d).

\section{DISCUSSION}

IGF2 has been identified as a major factor in the control of fetal growth in many species, including humans. IGF2 is a maternally imprinted gene, and its expression is regulated mostly by epigenetic marks. ${ }^{27}$ Epigenetic and genetic defects within 11p15.5 have been implicated in syndromes involving growth retardation (SRS, with IGF2 downregulation) ${ }^{28}$ or overgrowth (Beckwith-Wiedemann syndrome, with IGF2 overexpression). ${ }^{29}$ Furthermore, the overexpression of IGF2 is frequently observed in several types of tumors, including embryonal tumors in particular. ${ }^{30}$ The epigenetic regulation of IGF2 expression is well described, but the upstream genetic mechanism by which IGF2 expression is regulated remains unknown. 
N Table 1 Clinical features of patients with Silver-Russell syndrome and mutations of the PLAG1, HMGA2, or IGF2 genes

TeNe

Case

Defect

Familial case

Familial case

patient I-2

NM_002655.2:c.

439del

Frameshift- premature

Frameshi stop Deleterious

ClinVar accessions

Gender

Phenotype

Gestational age (weeks

Female

of amenorrhea)

\section{Birth length $\mathrm{cm} / \mathrm{SDS}$}

Birth weight $\mathrm{g} / \mathrm{SDS}$

Birth head circumference

$\mathrm{cm} / \mathrm{SDS}^{\mathrm{a}}$

Relative macrocephaly

at birth

$\mathrm{cm} / \mathrm{SDS}$

Weight $\mathrm{g}$ at 2 years/SDS

BMI at 2 years/SDS

Feeding difficulties during

infancy

Prominent forehead Yes

during infancy

Body asymmetry

$\mathrm{N}-\mathrm{H}$ score

Head circumference

$\mathrm{cm} / \mathrm{SDS} 2 \mathrm{yr}^{\mathrm{a}}$

Triangular facies

Final height $\mathrm{cm} / \mathrm{SDS}$

SRS adult

Term

Very small Very light

No

No

Familial case

PLAG1

HMGA2

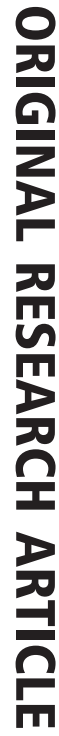

$\mathrm{GH}$ therapy and age at stat

Gl/feeding therapies No

Bsl IGF1 (ng/ml)/SDS ND

Bsl IGFBP3 (mg/L)

[R 0.8-3.0 M 2.1]/ALS/SDS

$\mathrm{GH}$ dose $(\mathrm{mg} / \mathrm{kg} / \mathrm{wk}) /$

Very small

$79 /-2.0$

$8,520 /-3.7$

$13.5 /-2.3$

Yes

Frameshift-premature patient II-2

NM_002655.2:c.

439del

Sporadic case

Sporadic case

Sporadic case

NM_003483.4:

C.193C > T

NM_003483.4

c.189del

$\begin{array}{ll}\text { Frameshift-premature } & \text { Frameshift-premature } \\ \text { stop Deleterious } & \text { stop Deleterious }\end{array}$

Premature stop

Frameshift-elong

protein Deleterious

SCV000297814

SCV00029781

Female

SRS 39 w 6

Female

$\begin{array}{ll}\text { Female } & \text { Fer } \\ \text { SRS } & \text { SRS }\end{array}$

Female

Female

44.5/-2.3 47/-2

$2,557 /-1.9$

$31 \mathrm{w} 6 \mathrm{~d}$

$37.5 /-2.78$

$1,050 /-3$

$27.4 /-1.5$

No

Yes

$75 /-2.8$

$79 /-2.0$

6,600/-6.7

$11.7 /-3.8$

Yes

7,800/-4.4

12.5/-3.2

Yes

Yes

Yes

No

No

$3 / 6$

$43.9 /-2.6$

No

$4 / 6$

$51.5 / S D S-2.8$

Yes Yes

Yes

NA

Yes: $2.5 \mathrm{yr}$

Yes: 2

Cyproheptadine

Cyproheptadine

$162 /+2.8$

$2.6 / 20 /+5.8$

67/-1.5

$2.5 / 10 /+1.8$

$0.26 / 368 /+7.2$

$0.27 / 195 /+3.1$

$3.5 / 19 /+4.5$

$3.0 / 16 . /+3.4$

$3.4 / 14 /+2.8$

$393^{d}$

$299^{d}$

No

$44.7 /-2.0$

$498^{\mathrm{d}}$

$\mathrm{Gl}$, ga

ND

43.5/-3.9

$2,300 /-2.5$

Yes

91.5/-3 (4 yr)

6,850/-3.6

Yes

$11,800 /-4(4 \mathrm{yr})$

14.1/-1.1

Yes

Yes

No

$5 / 6$

$44.5 /-1.2$

Yes

es

No

$5 / 6$

$46.4 /-1.7$

NA

Yes Yes

NA

Yes: $6 \mathrm{yr}$

NA

NGT $1.6 \mathrm{yr}$

NA

NA

Yes Yes

Yes: $3.3 \mathrm{yr} \quad$ Yes: $4.4 \mathrm{yr}$

Cyproheptadine $^{c} \quad$ NGT 2 months

$\begin{array}{llll}\text { ND } & 142 /+1.3 & 152 /+3.30 & 285 /+5.4\end{array}$

ND ND

ND $\quad 2.0 / N D / N D$

ND

NA

NA

$0.31 / 402 /+5$

$0.34 / 525 /+9.0$

$0.36 / 348 /+4.5$

IGF2 $(\mathrm{ng} / \mathrm{ml})$

SDS, standard deviation score: SRS, Silver-Russell syndrome.

The criteria of the Netchine-Harbinson scoring system are marked in bold characters.

aHead circumference at birth $\geq 1.5$ SDS above birth weight and/or length SDS. ${ }^{b}$ For this adult patient, the Netchine-Harbison clinical scoring system was determined from four criteria only, as the two additional items (birth

head circumference and BMl at 2 years) were not available. 'Cyproheptadine, a first-generation antihistamine,
assay, 3-7 years normal range $397-973 \mathrm{ng} / \mathrm{ml}$. 'EGF2 Esoterix assay, prepubertal normal range 334-642 ng/ml. 
a

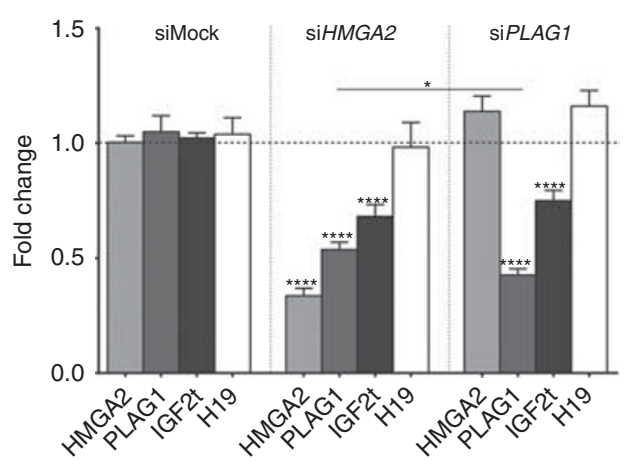

C

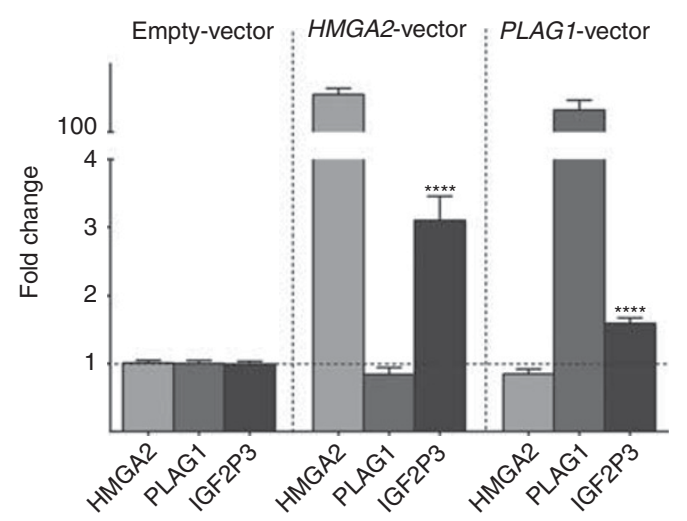

b

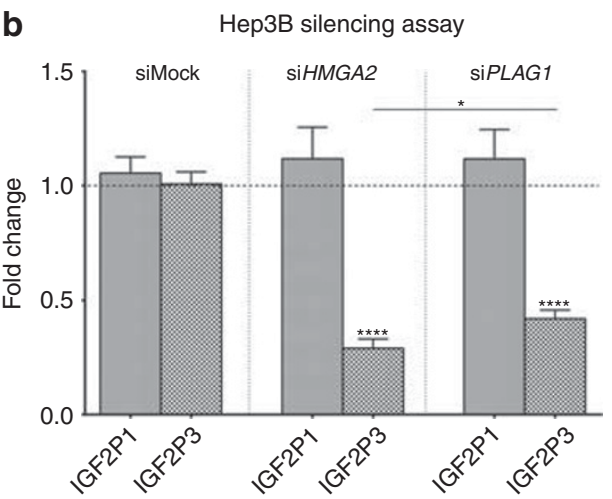

d

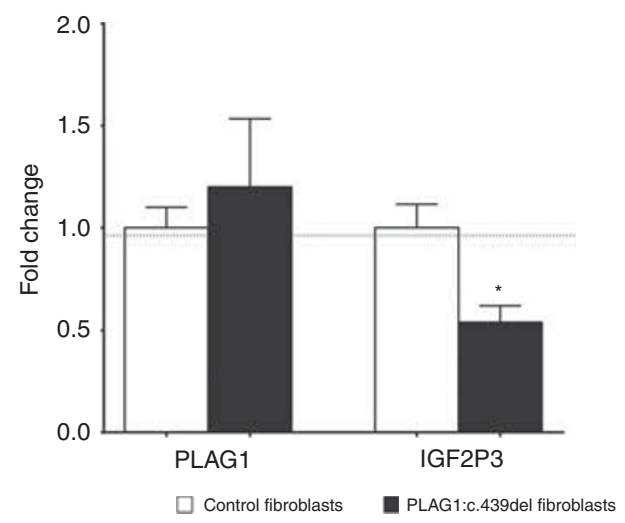

Figure 2 Functional characterization of the effects of HMGA2 and PLAG1 silencing/mutation on IGF2 expression. (a) HMGA2 is an upstream regulator of PLAG1, which in turn is an upstream regulator of IGF2. (b) The HMGA2-PLAG1 pathway regulates IGF2-P3 expression. (c) The IGF2 regulation by HMGA2 in a PLAG1-independent manner. (d) The effect of the PLAG1:c.439del on IGF2 expression in cultures of patient fibroblasts. A single asterisk indicates a $P<0.05$, and four asterisks indicate a $P<0.0001$. The histograms represent the fold change means of biological replicates. Three separate small interfering RNA (siRNA) and vector transfection assays were performed. Each transfection assay contained at least four transfected wells of control and siRNA or vector transfection $(N \geq 12$ biological sample per group per assay). For fibroblast expression, five cultures of control fibroblasts derived from different donors were compared to five different passages of cultured fibroblasts from the patient carrying the PLAG1 singlenucleotide deletion. The error bars indicate the standard error of the mean.

We report the first mutations of PLAG1 in a familial case with dominant transmission of syndromic IUGR associated with SRS and in another sporadic case of SRS with a de novo mutation. Since PLAG1 is a key factor in the HMGA2PLAG1-IGF2 pathway, we screened for and identified mutations of the HMGA2 and IGF2 genes. These findings highlight the crucial role of this pathway in controlling fetal IGF2 levels and its involvement in the pathophysiology of IUGR and SRS (Figure 3).

These observations also demonstrate that, in addition to epimutation at the $11 \mathrm{p} 15$ locus and mutation in the imprinted IGF2 gene, SRS may be caused by mutations of genes controlling IGF2 expression, such as PLAG1 and HMGA2. Though previously shown in mice, ${ }^{31}$ this is one of the first examples in humans, where nonimprinted genes deregulate the expression of distant, normally imprinted genes.

The mutations we identified in PLAG1 and HMGA2 were heterozygous leading to dominant transmission of SRS. The mutations we found in IGF2 were also heterozygous, but because IGF2 is paternally expressed in most tissues during fetal and postnatal development, to induce the SRS phenotype, an IGF2 mutation must occur on the paternal allele. We could not find any other inherited polymorphism near the de novo mutations in the IGF2 gene to specifically determine the parental origins of these mutations. However, given the typical SRS phenotype of these reported patients who carry the de novo IGF2 mutations, it is very likely that the mutations affected their paternal alleles, consistent with the imprinted status of this gene.

Very few mechanistic data on the HMGA2-PLAG1-IGF2 pathway or its role in pathological processes have been reported to date. All previous studies have shown HMGA2PLAG1 and PLAG1-IGF2 expression levels to be correlated, in overexpression models or in tumors. ${ }^{18,32}$ Murine models with inactivation of Hmga2, ${ }^{19}$ Plag1 ${ }^{17}$ or $\operatorname{Ig} 2^{33}$ display growth retardation, but the processes underlying the pathological phenotype have yet to be determined. 

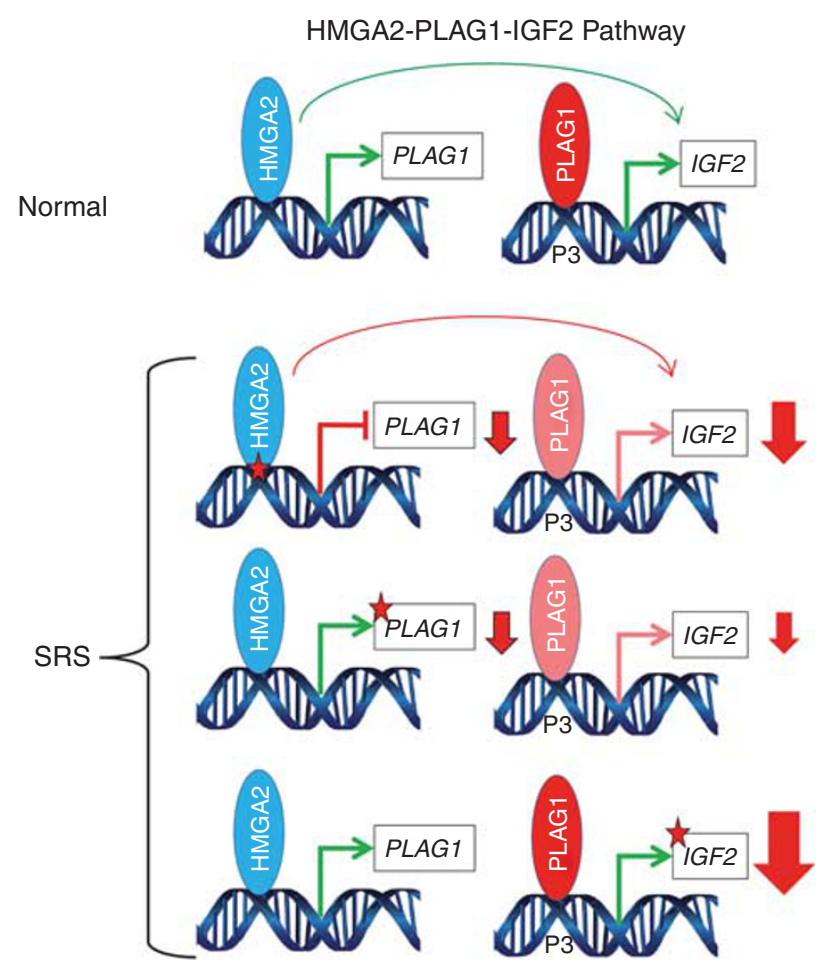

Figure 3 Schematic representation of the HMGA2-PLAG1-IGF2 pathway in a normal individual and in Silver-Russell cases. Green arrows indicate normal expression, the sizes of red arrows are representative of IGF2 downregulation and red stars indicate functional impairment for HMGA2, PLAG1, or IGF2 expression. SRS, Silver-Russell syndrome.

We show that the disruption of any genes in this pathway leads to a decrease in IGF2 expression and produces a SRS phenotype, similar to patients carrying 11 p15.5 epigenetic defects. We also show that this downregulation of IGF2 expression is caused by a lack of activation of the IGF2 P3 promoter, consistent with the results of previous studies showing a direct interaction between PLAG1 and this promoter. ${ }^{34}$ IGF2-P3 is the main transcript and is highly active in fetal tissues, whereas IGF2-P1 is active only in the liver after birth. ${ }^{35}$ This specific action at IGF2-P3 may account for the nondecreased levels of IGF2 in patients with mutations of the PLAG1 and HMGA2 genes. Indeed, circulating IGF2 is synthesized from the P1 promoter in a biallelic fashion by the adult liver in humans with no imprinting in this organ. ${ }^{36}$ The silencing of HMGA2 or PLAG1 had no effect on IGF2 transcription from the P1 promoter. Therefore, patients with an HMGA2-PLAG1 defect display conserved P1 transcription activity in the liver, resulting in normal postnatal IGF2 serum levels. The patients with the IGF2 mutations had low normal or modestly decreased levels of IGF2 in the serum (Table 1). In this case, the circulating IGF2 was probably produced by transcription from the unaffected allele alone. This is in accordance with the modestly decreased circulating IGF2 levels observed in the previously described patients carrying paternal $I G F 2^{10}$ mutations (Table 1).
As expected with a genetic defect, none of the cases have body asymmetry. In a family carrying this kind of mutation, body asymmetry is not expected to occur because the molecular defect is present in all the cells of the body (unlike the mosaic epigenetic change at the $11 \mathrm{p} 15.5$ locus). Thus, the absence of body asymmetry does not exclude the diagnosis of SRS in this particular case. However, despite the fact that patients harboring a genetic defect of the HMGA2-PLAG1-IGF2 pathway presented IUGR and an SRS clinical diagnosis, some differences were observed between the phenotypes. Indeed, patients with the IGF2 and HMGA2 defects were smaller at birth and their head circumferences were larger than those patients with PLAG1 defects. These differences between the phenotypes could simply reflect individual human variations, and more cases are needed to confirm these observations. Such variability is also observed within the group of SRS patients with IGF2/H19 hypomethylation. However, using siRNA-based silencing technique in cell lines, we showed that $H M G A 2$ silencing led to significantly lower levels of IGF2 expression than PLAG1 silencing, despite a more effective PLAG1 downregulation in the latter silencing assay. Furthermore, using vector-based overexpressing technique in another cell line, we showed that HMGA2 is capable of regulating IGF2 expression in a PLAG1-independent manner. Thus, IGF2 expression is more strongly affected by IGF2 and HMGA2 mutations, respectively, than by PLAG1 mutations.

The elucidation of these new genetic mechanisms has major implications for genetic counseling. In keeping with the imprinted, paternally expressed nature of the gene, males carrying IGF2 mutations have a 50\% risk of transmitting the disorder. As HMGA2 and PLAG1 are not imprinted, males and females with mutations of these genes both have a $50 \%$ risk of transmitting SRS. Given this high risk of SRS transmission, screening for mutations of these genes should be considered in cases of SRS presenting with no epigenetic defect of 11p15.5 or maternal disomy of chromosome 7. Furthermore, in the context of next-generation sequencing, these genes could be included in panels for the screening of growth retardation disorders for patients with no $11 \mathrm{p} 15$ epimutations or maternal disomy of chromosome 7 or 14 .

In conclusion, we show for the first time that defects of the HMGA2-PLAG1-IGF2 pathway can lead to SRS, highlighting the role of this oncogenic pathway in the fine regulation of physiological fetal/postnatal growth and food intake. We also show that nonimprinted genes can deregulate the expression of distant imprinted genes in humans. Given the crucial role played by the low level of IGF2 expression in the outcome of IUGR and the SRS phenotype, identification of new targets of IGF2 will be the next step in the development of new treatment options for this group of patients.

\section{Accession numbers}

The ClinVar accession numbers for the PLAG1, HMGA2, and $I G F 2$ variant sequences reported in this paper are listed in Table 1. 


\section{SUPPLEMENTARY MATERIAL}

Supplementary material is linked to the online version of the paper at http://www.nature.com/gim

\section{ACKNOWLEDGMENTS}

This work was supported by the Institut National de la Santé et de la Recherche Médicale, funding from the Université Pierre et Marie Curie (UPMC-Paris6), the Agence Nationale de la Recherche (ANR EPIFEGRO 2010) and from the "Association Françaises des Familles ayant un enfant atteint du Syndrome Silver Russell ou né Petit pour l'Age Gestationnel (AFIF/PAG)". W.A.H. was supported by the People Programme Marie Curie Actions (MCA) of the European Union's Seventh Framework Programme FP7/ITN Ingenium 20072013/ under REA grant agreement no. 290123 and by the Société Française d'Endocrinologie et Diabétologie Pédiatrique. F.B. was supported by a Novonordisk "Growth Hormone, Growth and Metabolism" grant. J.T.B. is supported by a Pediatric Early Research Career Award from Seattle Children's Research Institute. We received funding from the Fondation des Maladies Rares (France) for exome sequencing in the familial case. We thank the patients, their families, and their physicians (Jeanne Languepin, Marie-Pierre Cordier, and Noel Peretti), and the MAGIC Foundation, for their support. We also thank Françoise Praz from the Saint Antoine Research Centre (INSERM_U938) for kindly providing the Hep3B cell line for the silencing assays and Sandra ChantotBastaraud for performing the SNP arrays for the familial case. In addition, we are grateful to Laurence Perin for performing IGF2 determinations on patients' serum, and Nathalie Thibaut, Cristina Das Neves, Marilyne le Jule and Evelyne Tagodoe, the diagnostic technicians of the Pediatric Endocrinology Department, Trousseau Pediatric Hospital.

W.A.H., I.N., and Y.L.B. are members of the European Union's Seventh Framework Programme FP7/ITN Ingenium 2007-2013. F.B. and I.N. are members of the EUCID.net network COST (BM1208).

\section{AUTHOR CONTRIBUTIONS}

W.A.H, F.B, S.A, Y.L.B, M.D.H and I.N conceived the project and analyzed the data. W.A.H performed and designed experiments. T.Y performed DNA extraction and reviewed the manuscript. T.E, J.T.B, A.L.R, F.T, J.S and M.D.H provided patients' samples and recorded the clinical data. W.A.H, F.B, M.D.H and I.N wrote the manuscript.

\section{DISCLOSURE}

The authors declare no conflict of interest.

\section{REFERENCES}

1. Sheridan C. Intrauterine growth restriction-diagnosis and management. Aust Fam Physician. 2005;34:717-723.

2. Azzi S, Salem J, Thibaud N, et al. A prospective study validating a clinical scoring system and demonstrating phenotypical-genotypical correlations in Silver-Russell syndrome. J Med Genet. 2015;52:446-453.

3. Dias RP, Nightingale $P$, Hardy $C$, et al. Comparison of the clinical scoring systems in Silver-Russell syndrome and development of modified diagnostic criteria to guide molecular genetic testing. J Med Genet. 2013;50:635-639.
4. Bartholdi D, Krajewska-Walasek M, Ounap K, et al. Epigenetic mutations of the imprinted IGF2-H19 domain in Silver-Russell syndrome (SRS): results from a large cohort of patients with SRS and SRS-like phenotypes. J Med Genet. 2009;46:192-197.

5. Netchine I, Rossignol S, Dufourg MN, et al. 11p15 imprinting center region 1 loss of methylation is a common and specific cause of typical Russell-Silver syndrome: clinical scoring system and epigeneticphenotypic correlations. J Clin Endocrinol Metab. 2007;92:3148-3154.

6. Wakeling EL, Brioude F, Lokulo-Sodipe O, et al. Diagnosis and management of Silver-Russell syndrome: first international consensus statement. Nat Rev Endocrinol. 2016;13:105-124.

7. Gicquel C, Rossignol S, Cabrol S, et al. Epimutation of the telomeric imprinting center region on chromosome $11 \mathrm{p} 15$ in Silver-Russell syndrome. Nat Genet. 2005;37:1003-1007.

8. Azzi S, Blaise A, Steunou V, et al. Complex tissue-specific epigenotypes in Russell-Silver Syndrome associated with 11p15 ICR1 hypomethylation. Hum Mutat. 2014;35:1211-1220.

9. Brioude $F$, Oliver-Petit I, Blaise A, et al. CDKN1C mutation affecting the PCNA-binding domain as a cause of familial Russell Silver syndrome. J Med Genet. 2013;50:823-830.

10. Begemann M, Zirn B, Santen G, et al. Paternally inherited IGF2 mutation and growth restriction. N Engl J Med. 2015;373:349-356.

11. Kas K, Voz ML, Roijer E, et al. Promoter swapping between the genes for a novel zinc finger protein and beta-catenin in pleiomorphic adenomas with $t(3 ; 8)(p 21 ; q 12)$ translocations. Nat Genet. 1997;15:170-174.

12. Astrom A, D'Amore ES, Sainati $L$, et al. Evidence of involvement of the PLAG1 gene in lipoblastomas. Int J Oncol. 2000;16:1107-1110.

13. Zatkova A, Rouillard JM, Hartmann W, et al. Amplification and overexpression of the IGF2 regulator PLAG1 in hepatoblastoma. Genes Chromosomes Cancer. 2004;39:126-137.

14. Voz ML, Agten NS, Van de Ven WJ, Kas K. PLAG1, the main translocation target in pleomorphic adenoma of the salivary glands, is a positive regulator of IGF-II. Cancer Res. 2000;60:106-113.

15. Hensen K, Van Valckenborgh IC, Kas K, Van de Ven WJ, Voz ML. The tumorigenic diversity of the three PLAG family members is associated with different DNA binding capacities. Cancer Res. 2002;62:1510-1517.

16. Declercq J, Van Dyck F, Braem CV, et al. Salivary gland tumors in transgenic mice with targeted PLAG1 proto-oncogene overexpression. Cancer Res. 2005;65:4544-4553.

17. Hensen K, Braem C, Declercq J, et al. Targeted disruption of the murine Plag1 proto-oncogene causes growth retardation and reduced fertility. Dev Growth Differ. 2004;46:459-470.

18. Klemke M, Muller MH, Wosniok W, et al. Correlated expression of HMGA2 and PLAG1 in thyroid tumors, uterine leiomyomas and experimental models. PLoS One. 2014;9:e88126.

19. Zhou X, Benson KF, Ashar HR, Chada K. Mutation responsible for the mouse pygmy phenotype in the developmentally regulated factor HMGIC. Nature. 1995;376:771-774.

20. De Crescenzo A, Citro V, Freschi A, et al. A splicing mutation of the HMGA2 gene is associated with Silver-Russell syndrome phenotype. J Hum Genet. 2015;60:287-293.

21. Fokstuen $S$, Kotzot D. Chromosomal rearrangements in patients with clinical features of Silver-Russell syndrome. Am J Med Genet $A$. 2014:164A:1595-1605.

22. Okada Y, Kamatani Y, Takahashi A, et al. A genome-wide association study in 19633 Japanese subjects identified LHX3-QSOX2 and IGF1 as adult height loci. Hum Mol Genet. 2010;19:2303-2312.

23. Cho YS, Go MJ, Kim YJ, et al. A large-scale genome-wide association study of Asian populations uncovers genetic factors influencing eight quantitative traits. Nat Genet. 2009;41:527-534.

24. Gudbjartsson DF, Walters GB, Thorleifsson G, et al. Many sequence variants affecting diversity of adult human height. Nat Genet. 2008;40: 609-615.

25. Weedon MN, Lettre G, Freathy RM, et al. A common variant of HMGA2 is associated with adult and childhood height in the general population. Nat Genet. 2007;39:1245-1250.

26. Chinuck R, Dewar J, Baldwin DR, Hendron E. Appetite stimulants for people with cystic fibrosis. Cochrane Database Syst Rev. 2014: CD008190.

27. Chao W, D'Amore PA. IGF2: epigenetic regulation and role in development and disease. Cytokine Growth Factor Rev. 2008;19:111-120.

28. Abi Habib W, Brioude F, Azzi S, et al. 11p15 ICR1 partial deletions associated with IGF2/H19 DMR hypomethylation and Silver-Russell syndrome. Hum Mutat. 2016;38:105-111. 
29. Abi Habib W, Azzi S, Brioude F, et al. Extensive investigation of the IGF2/ H19 imprinting control region reveals novel OCT4/SOX2 binding site defects associated with specific methylation patterns in BeckwithWiedemann syndrome. Hum Mol Genet. 2014;23:5763-5773.

30. Azzi S, Abi Habib W, Netchine I. Beckwith-Wiedemann and Russell-Silver Syndromes: from new molecular insights to the comprehension of imprinting regulation. Curr Opin Endocrinol Diabetes Obes. 2014;21: 30-38.

31. Mott R, Yuan W, Kaisaki $P$, et al. The architecture of parent-of-origin effects in mice. Cell. 2014;156:332-342.

32. Juma AR, Damdimopoulou PE, Grommen SV, Van de Ven WJ, De Groef B. Emerging role of PLAG1 as a regulator of growth and reproduction. J Endocrinol. 2016;228:R45-56.

33. DeChiara TM, Efstratiadis A, Robertson EJ. A growth-deficiency phenotype in heterozygous mice carrying an insulin-like growth factor II gene disrupted by targeting. Nature. 1990;345:78-80.

34. Akhtar M, Holmgren C, Gondor A, et al. Cell type and context-specific function of PLAG1 for IGF2 P3 promoter activity. Int J Oncol. 2012;41: 1959-1966.

35. Li X, Nong Z, Ekstrom C, et al. Disrupted IGF2 promoter control by silencing of promoter P1 in human hepatocellular carcinoma. Cancer Res. 1997;57:2048-2054.
36. Ekstrom TJ, Cui H, Li X, Ohlsson R. Promoter-specific IGF2 imprinting status and its plasticity during human liver development. Development. 1995;121:309-316.

37. Gnirke A, Melnikov A, Maguire J, et al. Solution hybrid selection with ultra-long oligonucleotides for massively parallel targeted sequencing. Nat Biotechnol. 2009;27:182-189.

\section{This work is licensed under a Creative Commons}

Attribution-NonCommercial-NoDerivs

International License. The images or other third party material in this article are included in the article's Creative Commons license, unless indicated otherwise in the credit line; if the material is not included under the Creative Commons license, users will need to obtain permission from the license holder to reproduce the material. To view a copy of this license, visit http://creativecommons.org/licenses/ by-nc-nd/4.0/

(C) The Author(s) 2018 